\title{
Restoration of
}

\section{Buildings and Monuments}

\section{Materials Science, Conservation of Architectural Heritage, Sustainable Construction}

\section{Editor-in-Chief}

Özlem Cizer, Leuven, Belgium

\section{Editorial Board}

Toshiki Ayano, Okayama, Japan

Luigia Binda, Milan, Italy

A. Elena Charola, Washington, DC, USA

Thierry Chaussadent, Champs-sur-Marne, France

Luc Courard, Liège, Belgium

Jian-Guo Dai, Hong Kong, China

Hilde De Clercq, Brussels, Belgium

José Delgado Rodrigues, Lisbon, Portugal

David W. Fowler, Austin, TX, USA

Mariaenrica Frigione, Salento, Italy

Andrzej Garbacz, Warsaw, Poland

Klaus Littmann, Hannover, Germany

Barbara Lubelli, Delft, Netherlands

Elisabeth Marie-Victoire, Paris, France

Bernhard Middendorf, Kassel, Germany

Michael Raupach, Aachen, Germany

Carlos Rodriguez Navarro, Granada, Spain

Johan Silfwerbrand, Stockholm, Sweden

Maria Rosa Valluzzi, Padova, Italy

Koen Van Balen, Leuven, Belgium

Dionys Van Gemert, Leuven, Belgium

Oliver Weichold, Aachen, Germany

Folker H. Wittmann, Honorary member, Freiburg, Germany 
Abstracted/Indexed In AATA Online: Abstracts of International Conservation Literature, USA; Getty Institute; Conservation Abstracts, Los Angeles, USA; Cambridge Scientific Abstracts, Beachwood, Ohio, USA; Institut national du patrimoine (inp), Saint-Denis La Plaine, France; ICONDA, The International Construction Database, Fraunhofer-Informationszentrum Raum und Bau IRB, Stuttgart, Germany

ISSN $1864-7251 \bullet$ e-ISSN $1864-7022$

All information regarding notes for contributors, subscriptions, open access, back volumes and orders is available online at www.birkhauser.com/rbm

Editor-In-Chief Prof. Özlem Cizer, KU Leuven, Department of Civil Engineering, Building Materials and Building Technology Section, Belgium

Journal Editor Alexander Felix, Birkhäuser Verlag GmbH, Allschwilerstrasse 10, 4055 Basel, Switzerland. Tel.: +41 (0)61 3061704 , Fax: +41 (0)61 30617 01, Email: RBM.editorial@birkhauser.ch

Journal Production Editor Esther Markus, De Gruyter, Genthiner Straße 13, 10785 Berlin, Germany, Tel.: +49 (0)30 26005 - 127 , Fax: +49(0)30 26005 - 250, Email: esther.markus@degruyter.com

Responsible For Advertisements Claudia Neumann, De Gruyter, Genthiner Straße 13, 10785 Berlin, Germany, Tel.: +49 (0)30 26005 - 226 Fax: +49 (0) 30.26005 - 264 Email: anzeigen@degruyter.com

(C) 2018 Birkhäuser Verlag GmbH, Basel

P.O. Box 44, 4009 Basel, Switzerland

Part of Walter de Gruyter GmbH, Berlin/Boston

Typesetting Integra Software Services Pvt. Ltd., Pondicherry, India

Printing Franz X. Stückle Druck und Verlag e.K., Ettenheim

Cover Illustration Micrographs of impregnated sandstones. Image courtesy of Franziska Braun, Jeanette Orlowsky: Department of Building Materials, Technische Universität Dortmund

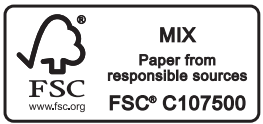

Offenlegung der Inhaber und Beteiligungsverhältnisse gem. § 7a Abs. 1 Ziff. 1, Abs. 2 Ziff. 3 des Berliner Pressegesetzes: Die Gesellschafter der Walter de Gruyter GmbH sind: Cram, Gisela, Rentnerin, Berlin; Cram, Elsbeth, Pensionärin, Rosengarten-Alvesen; Cram, Dr. Georg-Martin, Unternehmens-Systemberater, Stadtbergen; Cram, Maike, Wien (Österreich); Cram, Jens, Mannheim; Cram, Ingrid, Betriebsleiterin, Tuxpan/ Michoacan (Mexiko); Cram, Sabina, Mexico, DF (Mexiko); Cram, Silke, Wissenschaftlerin, Mexico DF (Mexiko); Cram, Björn, Aachen; Cram, Berit, Hamm; Cram-Gomez, Susana, Mexico DF (Mexiko); Cram-Heydrich, Walter, Mexico DF (Mexico); Cram-Heydrich, Kurt, Angestellter, Mexico DF (Mexico); Duvenbeck, Birgitta, Oberstudienrätin i.R., Bad Homburg; Gädeke, Gudula, M.A., Atemtherapeutin/Lehrerin, Tübingen; Gädeke, Martin, Einzelunternehmer, Ingolstadt; Lubasch, Dr. Annette, Ärztin, Berlin; Schütz, Dr. Christa, Ärztin, Mannheim; Schütz, Sonja, Berlin; Schütz, Juliane, Berlin; Schütz, Antje, Berlin; Schütz, Valentin, Mannheim; Seils, Dorothee, Apothekerin, Stuttgart; Seils, Dr. Ernst-Albert, Pensionär, Reppenstedt; Seils, Gabriele, Dozentin, Berlin; Seils, Christoph, Journalist, Berlin; Siebert, John-Walter, Pfarrer, Oberstenfeld; Tran, Renate, Mediatorin, Zürich (Schweiz). 


\section{Contents}

Franziska Braun and Jeanette Orlowsky Effect of Different Silicic Acid Ester on the Properties of Sandstones with Varying Binders - 1

Dirk HR Spennemann, Melissa Pike and Maggie J Watson Behaviour of Pigeon Excreta on Masonry Surfaces — 15
Dionys A. Van Gemert, Luc R. Taerwe and Kristof P. Verreydt Comparison of Evolution of Pop-Out Damage by Mixed in Burnt Lime Nodules in Precast Concrete in Outside and Inside Exposure Conditions - 29 